OPEN ACCESS

Edited by:

Paul Bogdan,

University of Southern California,

United States

Reviewed by:

Luigi Fortuna,

University of Catania, Italy

Peter Herman,

Yale University, United States

${ }^{*}$ Correspondence:

Sou Nobukawa

nobukawa@cs.it-chiba.ac.jp

tThese authors have contributed equally to this work

Specialty section:

This article was submitted to

Fractal and Network Physiology,

a section of the journal

Frontiers in Physiology

Received: 28 October 2020

Accepted: 19 January 2021

Published: 11 February 2021

Citation:

Nobukawa S, Shirama A, Takahashi T,

Takeda T, Ohta H, Kikuchi M,

Iwanami A, Kato N and Toda S (2021)

Pupillometric Complexity and

Symmetricity Follow Inverted-U

Curves Against Baseline Diameter Due to Crossed Locus Coeruleus Projections to the Edinger-Westphal

Nucleus. Front. Physiol. 12:614479.

doi: 10.3389/fphys.2021.614479

\section{Pupillometric Complexity and Symmetricity Follow Inverted-U Curves Against Baseline Diameter Due to Crossed Locus Coeruleus Projections to the Edinger-Westphal Nucleus}

\author{
Sou Nobukawa ${ }^{1 * t}$, Aya Shirama ${ }^{2+}$, Tetsuya Takahashi ${ }^{3,4,5 t}$, Toshinobu Takeda ${ }^{6}$, \\ Haruhisa Ohta ${ }^{7}$, Mitsuru Kikuchi ${ }^{8}$, Akira Iwanami ${ }^{9}$, Nobumasa Kato ${ }^{7}$ and \\ Shigenobu Toda ${ }^{8,10+}$
}

\begin{abstract}
${ }^{1}$ Department of Computer Science, Chiba Institute of Technology, Chiba, Japan, ${ }^{2}$ National Center of Neurology and Psychiatry, Department of Preventive Intervention for Psychiatric Disorders, National Institute of Mental Health, Tokyo, Japan, ${ }^{3}$ Research Center for Child Mental Development, Kanazawa University, Ishikawa, Japan, ${ }^{4}$ Department of Neuropsychiatry, University of Fukui, Fukui, Japan, ${ }^{5}$ Uozu Shinkei Sanatorium, Uozu, Japan, ${ }^{6}$ Faculty of Letters, Ryukoku University, Kyoto, Japan, ${ }^{7}$ Medical Institute of Developmental Disabilities Research, Showa University, Tokyo, Japan, ${ }^{8}$ Department of Psychiatry \& Behavioral Science, Kanazawa University, Ishikawa, Japan, ${ }^{9}$ Department of Psychiatry, School of Medicine, Showa University, Tokyo, Japan, ${ }^{10}$ Department of Psychiatry, Showa University East Hospital, Showa University, Tokyo, Japan
\end{abstract}

In addition to photic reflex function, the temporal behavior of the pupil diameter reflects levels of arousal and attention and thus internal cognitive neural activity. Recent studies have reported that these behaviors are characterized by baseline activity, temporal complexity, and symmetricity (i.e., degree of symmetry) between the right and left pupil diameters. We hypothesized that experimental analysis to reveal relationships among these characteristics and model-based analysis focusing on the newly discovered contralateral projection from the locus coeruleus (LC) to the Edinger-Westphal nucleus $(E W N)$ within the neural system for controlling pupil diameter could contribute to another dimension of understanding of complex pupil dynamics. In this study, we aimed to validate our hypothesis by analyzing the pupillary hippus in the healthy resting state in terms of sample entropy (SampEn), to capture complexity, and transfer entropy (TranEn), to capture symmetricity. We also constructed a neural model embedded with the new findings on neural pathways. The following results were observed: first, according to surrogate data analysis, the complexity and symmetricity of pupil diameter changes reflect a non-linear deterministic process. Second, both the complexity and the symmetricity are unimodal, peaking at intermediate pupil diameters. Third, according to simulation results, the neural network that controls pupil diameter has an inverted U-shaped profile of complexity and symmetricity vs. baseline LC activity; this tendency is enhanced by the contralateral synaptic projections from the LCs to the EWNs. Thus, we characterized the typical relationships between the baseline activity and the 
complexity and symmetricity of the pupillometric data in terms of SampEn and TranEn. Our evaluation method and findings may facilitate the development of estimation and diagnostic tools for exploring states of the healthy brain and psychiatric disorders based on measurements of pupil diameter.

Keywords: pupillometry, complexity, symmetricity, neural model, non-linear dynamics, hippus

\section{INTRODUCTION}

Over decades, studies of pupil-diameter behavior (pupillometry) have revealed that it reflects internal neural activity affecting arousal and attention, in addition to simple photic reflexes (Rajkowski, 1993; Gilzenrat et al., 2003; Nassar et al., 2012; Eldar et al., 2013; Ebitz et al., 2014; Hong et al., 2014; Reimer et al., 2014) (review in Aston-Jones and Cohen, 2005; McGinley et al., 2015; Szabadi, 2018). The reason is that the locus coeruleus (LC), which is the norepinephrine (NE) source for both the sympathetic pathway to the pupil dilator muscle and the parasympathetic pathway to the pupil sphincter muscle, also modulates cognitive functions (Breen et al., 1983; McCormick and Pape, 1990; Ego-Stengel et al., 2002; Hirata et al., 2006; Moxon et al., 2007; Reimer et al., 2014; Martins and Froemke, 2015; McGinley et al., 2015; Wekselblatt and Niell, 2015). The LC adjusts the exploration-exploitation tradeoff in the brain by its activity pattern, i.e., it has a tonic firing mode corresponding to the exploration process (LC activity occurs for a long term and is continuous, with a high baseline activity of 1-5 Hz) and a phasic firing mode corresponding to the exploitation process (LC activity is sparse and bursty with a low baseline activity; review in Aston-Jones and Cohen, 2005). The baseline LC activity in these firing modes is strongly reflected in pupil diameter (Rajkowski, 1993; Gilzenrat et al., 2003; Joshi et al., 2016) (review in Aston-Jones and Cohen, 2005). Several experimental studies of pupil diameter in humans and mouse models show that it represents cognitive processes in both the healthy brain (reviewed in Aston-Jones and Cohen, 2005; van der Wel and van Steenbergen, 2018) and psychiatric disorders. In the latter, pupil diameter can reflect deficits of attention or arousal or imbalances in the exploration-exploitation tradeoff, which are observed in schizophrenia (Reddy et al., 2018; Thakkar et al., 2018), autism spectrum disorder (ASD), (Anderson and Colombo, 2009; Martineau et al., 2011; Gotham et al., 2018; Bast et al., 2019), and attention-deficit hyperactivity disorder (ADHD) (Wainstein et al., 2017).

Another approach to the study of pupil behavior is mathematical modeling. Various neural models have been constructed with the aim of reproducing pupil behavior in the resting state and under various types of visual input (Usui and Stark, 1982; Longtin and Milton, 1989; Pamplona et al., 2009; Watson and Yellott, 2012; Johansson and Balkenius, 2018). Usui and Stark first described the characteristic of temporal complexity in a modeling study of the autonomous fluctuation of pupil diameter called hippus (Usui and Stark, 1982). In their model, internal fluctuating neural activity is applied to the sympathetic pathway to the pupil dilator muscle and to the parasympathetic pathway to the pupil sphincter muscle. They showed that temporal complexity as measured by the standard deviation of pupil diameter exhibits an inverted U-shape against pupil diameter. This effect results from the non-linear relationship between pupil dilation and the applied neural activity. This profile of complexity is highly congruent with experimental findings (Usui and Stark, 1982). Johansson and Balkenius constructed a more complex model of pupil control based on a neural system comprising amygdala, LC, cerebellum, and other regions (Johansson and Balkenius, 2018). Interactions among neural activities, especially cortical activities, among farflung brain regions could reproduce several kinds of reactions of pupil diameter to stimuli and exhibit emotional and learning effects (Johansson and Balkenius, 2018).

Furthermore, recent progress in research on pupil diameter has provided new insights into the temporal patterns of pupil diameter and its controlling neural pathways (Liu et al., 2017; Poynter, 2017; Wahn et al., 2017; Artoni et al., 2019; Nakamura et al., 2019; Piu et al., 2019). In particular, it has been discovered that the parasympathetic pathways from the LC project are inhibitory to both the ipsilateral and contralateral parts of the Edinger-Westphal nucleus (EWN) to control the pupil sphincter muscles, not only to the ipsilateral parts as previously thought (Liu et al., 2017). Nakamura et al. and Artoni et al. demonstrated that the temporal hippus patterns reflect several stages of cognitive processing (Nakamura et al., 2019) and are affected by psychiatric disorders (Artoni et al., 2019). Piu et al. showed that the hippus fluctuation reflects the non-linear dynamics of autonomous neural activity; this determinism and non-linear complexity might be utilized as a diagnostic tool for detecting the states of neural systems (Piu et al., 2019). In addition to the temporal pattern of pupil diameter, the asymmetricity between the right and left pupil diameters is informative, reflecting the attention function (Poynter, 2017; Wahn et al., 2017). In particular, Wahn et al. showed that this asymmetricity exhibits dependency on attentional load and events (Wahn et al., 2017). Poynter reported that pupil asymmetricity reflects gender, attentional load, and symptoms of ADHD such as inattention and hyperactivity (Poynter, 2017). However, the mechanisms that induce complex temporal patterns and asymmetry in pupil diameters remain unclear.

A model-based approach, which can describe internal neural activity as arising from interactions among brain regions and non-linear neural characteristics, and the approach of combining modeling with experimentation, are effective in revealing the mechanisms of complexity and asymmetricity in pupil behavior. We hypothesized that a model-based approach focusing on the newly discovered contralateral projection from the LC to the 
EWN combined with an experimental study of the relationship between complexity and asymmetricity would be effective in elucidating the complexity of pupil behavior. In the present study, we aimed to validate our hypothesis by analyzing the hippus in the healthy resting state and constructing a neural model embedded with the new findings on neural pathways. Concretely, we analyze the relationships between baseline pupil activity during hippus and sample entropy (SampEn) (Richman and Moorman, 2000), a measure of temporal complexity, and transfer entropy (TranEn) (Schreiber, 2000), a measure of the asymmetricity of the left and right pupil diameters. Furthermore, by constructing a neural system for controlling pupil diameter that incorporates the contralateral projections to the EWN from the LC, the influences of these projections on temporal complexity and asymmetricity were evaluated.

\section{MATERIALS AND METHODS}

\subsection{Participants}

The study was performed at the Medical Institute of Developmental Disabilities Research, Showa University, Japan, and included 17 healthy participants ( 7 male, 10 female, average age $36.3 \mathrm{y}, \mathrm{SD}=8.0$, range $=22-51$ ). The exclusion criteria were as follows: a current major depressive or manicdepressive episode, a history of psychosis, Wechsler full-scale intelligence quotient $<80$, a history of head injury with loss of consciousness, a sensory-motor handicap, or neurological illness. All participants had normal or corrected-to-normal vision and normal hearing. After providing complete explanation of the study procedures, written informed consent was obtained. All methods were performed in accordance with the Declaration of Helsinki, and the study protocol was approved by the Ethics Committee of Showa University.

\subsection{Recording Pupil Diameters}

Participants did not use caffeine, nicotine, or any medication that might influence eye movements on the day of the experiment. For the measurements of pupil diameter, the participants sat in front of a monitor subtending $50.9 \times 28.6$ degrees of visual angle at a distance of $57 \mathrm{~cm}$, in a lit room. The participant's head position was fixed by a chin rest. To obtain the pupil-diameter data during hippus, the participants were instructed to gaze steadily at a fixation point consisting of a black cross displayed on a screen of brightness $0.87 \mathrm{~cd} / \mathrm{m}^{2}$ for $2 \mathrm{~min}$. The cross subtended $0.5 \times 0.5$ degrees of visual angle. The fixation points were generated with the Psychophysics Toolbox routines (Brainard, 1997; Pelli, 1997) for MATLAB (Version 2013b, MathWorks Ltd, http://www.mathworks.com/) and presented on a 23-inch LCD monitor $(1920 \times 1080$ pixels at $60 \mathrm{~Hz})$ driven by a PC (Windows $7)$. During measurements, the participant's eye positions and pupil diameters were simultaneously recorded by a remote-type eye tracker (TX300; Tobii Technology, Stockholm, Sweden) with a sampling frequency of $300 \mathrm{~Hz}$. In Figure 1, typical time-series of pupil diameters was shown.

In the time-series, the unmeasurable periods appeared due to the blinks and eye movements. Because the time-series in these periods disturbed the results of evaluation indexes, we chose the periods not including unmeasurable periods as much as possible by setting a short epoch length. Therefore, at the preprocessing stage, the time-series data were divided into epochs of length $2.0 \mathrm{~s}$ and missing values were linearly interpolated. Epochs missing more than $10 \%$ of epoch length were excluded from the analysis. Evaluations of size, complexity, and symmetricity are independently conducted at each epoch. The epochs were low-pass filtered between 0 and $50 \mathrm{~Hz}$. At the end of the preprocessing stage, the epoch length is $2 \mathrm{~s}$, consisting of 600 data points. The number of useable epochs per participant was average, 39.7; SD, 17.4; and range, 5-67.

\subsection{Pupil-Diameter Analysis: Complexity}

To quantify the temporal complexity of pupil diameter, we calculated the SampEn. From $N$ stochastic variables $\left\{x_{1}, x_{2}, \cdots x_{N}\right\}$ normalized by $z$-score, $m$-dimensional vectors are constructed:

$$
\mathbf{x}_{i}^{m}=\left\{x_{i}, x_{i+1}, \cdots, x_{i+m-1}\right\},
$$

The probability $C_{m}(r)$ is calculated over $i, j(i \neq j, i, j=1,2, \cdots)$ pairs of these vectors as follows:

$$
C_{m}(r)=\sum_{i, j \in r, i \neq j} \frac{\left|\mathbf{x}_{i}^{m}-\mathbf{x}_{j}^{m}\right|}{(N-m+1)(N-m)} .
$$

where $\left|\mathbf{x}_{i}^{m}-\mathbf{x}_{j}^{m}\right|$ indicates that it is counted when the distance between any two vectors $\mathbf{x}_{i}^{m}$ and $\mathbf{x}_{j}^{m}$ as the norm is less than $r$. SampEn is then defined as

$$
h(r, m)=-\log \frac{C_{m+1}(r)}{C_{m}(r)} .
$$

In this study, we used $r=0.2, m=2$ (Costa et al., 2002). $h(r, m)$ increases monotonically with the complexity of the time series. In calculating the SampEn, we used PhysioToolkit at the following address: http://physionet.incor.usp.br/physiotools/sampen/ as a toolbox in MATLAB ${ }^{\circledR}$ (Goldberger et al., 2000).

\subsection{Pupil-Diameter Analysis: Symmetricity}

To evaluate the symmetricity of the right and left pupil diameters, we used the causality between the right and left pupil diameters as captured by the TranEn. The TranEn $T_{X \rightarrow Y}$ from the timeseries $\left\{x_{1}, x_{2}, \cdots, x_{t}, \cdots\right\}$ to the time-series $\left\{y_{1}, y_{2}, \cdots, y_{t}, \cdots\right\}$ is defined as follows (Schreiber, 2000):

$$
T_{X \rightarrow Y}=\sum_{y_{t+\tau}, \mathbf{y}_{\mathbf{t}}{ }^{d_{y}}, \mathbf{x}_{\mathbf{t}} d_{x}} p\left(y_{t+\tau} \mid \mathbf{y}_{\mathbf{t}}{ }^{d_{y}}, \mathbf{x}_{\mathbf{t}}{ }^{d_{x}}\right) \log \left(\frac{p\left(y_{t+\tau} \mid \mathbf{y}_{\mathbf{t}}{ }^{d_{y}}, \mathbf{x}_{\mathbf{t}}{ }^{d_{x}}\right)}{p\left(y_{t+\tau} \mid \mathbf{y}_{\mathbf{t}}{ }^{d_{y}}\right)}\right) .
$$

where $t$ is the time index; $p(\cdot \mid \cdot)$ is the conditional probability. $\mathbf{y}_{\mathbf{t}} d_{y}$ and $\mathbf{x}_{\mathbf{t}} d_{x}$ denote $d_{x}$ - and $d_{y}$-dimensional delay vectors:

$$
\begin{aligned}
& \mathbf{x}_{\mathbf{t}}{ }^{d_{x}}=\left(x_{t}, x_{t-\tau}, \cdots, x_{t-\left(d_{x}-1\right) \tau}\right), \\
& \mathbf{y}_{\mathbf{t}}{ }^{d_{y}}=\left(y_{t}, y_{t-\tau}, \cdots, y_{t-\left(d_{y}-1\right) \tau}\right) .
\end{aligned}
$$




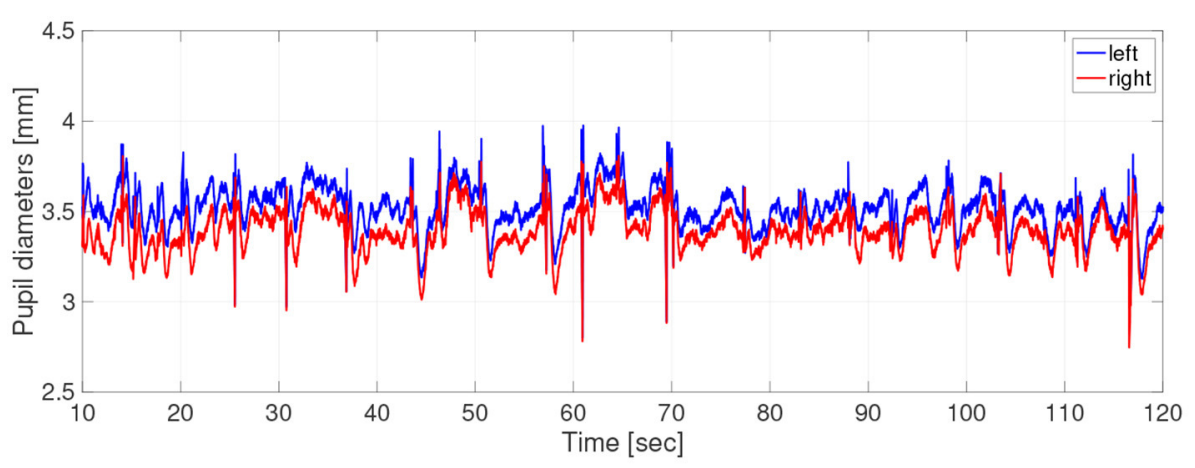

FIGURE 1 | Typical example of time-series of pupil-diameters. Here, missing values due to blinks and eye movements were linearly interpolated; the low-pass filtered between 0 and $50 \mathrm{~Hz}$ was applied.

We used $\tau=10$ (corresponding to a period of $0.033 \mathrm{~s}$ ), $d_{x}=5$, and $d_{y}=5$. If there is no causality from $X$ to $Y, T_{X \rightarrow Y}=0$. With increasing causality from $X$ to $Y, T_{X \rightarrow Y}$ becomes larger. In this study, TranEn from the right to left pupils and that from the left to right pupils were measured. High TranEn $(\gg 0)$ indicates high symmetricity between the pupil diameters, while low TranEn $(\approx 0)$ indicates the emergence of asymmetricity. In calculating the TranEn, we used HERMES as a toolbox in MATLAB ${ }^{\circledR}$ (Niso et al., 2013).

\subsection{Neural Model of the Controller of Pupil Diameter}

Figure 2 shows our model of the neural pathways that control pupil diameter, which includes the contralateral projections from the LC to the EWN discovered by Liu et al. (2017). The LC consists of multiple synchronously firing neuron ensembles, not one globally synchronous neural population (reviewed in Totah et al., 2019). Therefore, in this study, we assumed different neural populations in the left and right LCs. Figure 3 shows an overview of the model as a methodological flowchart of the neural model of the controller of pupil diameter. To describe the LC neuronal population activity, we utilized the coupled Lorenz models $X_{i}, Y_{i}, Z_{i}(i=1,2)$ as follows:

$$
\begin{gathered}
\frac{d X_{i}}{d t}=a_{i}\left(Y_{i}-X_{i}\right)+J\left(X_{j}-X_{i}\right) \\
\frac{d Y_{i}}{d t}=c_{i} X_{i}-X_{i} Z_{i}-Y_{i} \\
\frac{d Z_{i}}{d t}=X_{i} Y_{i}-b_{i} Z_{i}
\end{gathered}
$$

where, $X_{i}, Y_{i}, Z_{i}$ represent the variable of Lorenz models coupled by strength of $J$. The index $i$ distinguishes the left and right sides. We also assumed that the neural activities in the left and right LC weakly synchronize with each other. Therefore, we used the parameter set $\left(a_{i}=10, b_{i}=8 / 3, c_{i}=28, J=0.7\right)$ to produce weakly synchronized state (correlation coefficient between $X_{1,2}$ : $R \approx 0.165)$. The initial values $\left(X_{1}, Y_{1}, Z_{1}\right)=(0.0,1.0,1.0)$ and
$\left(X_{2}, Y_{2}, Z_{2}\right)=(0.0,1.1,1.1)$ were used. The LC neural population activities $x_{i}(i=1,2)$ are then given by

$$
x_{i}=L \operatorname{zscore}\left(X_{i}\right)+b_{i},
$$

where $L$ and $b_{i}$ are parameters. $b_{i}$ is the LC baseline firing rate. In this study, $b_{1}=b_{2}=b$. zscore indicates the application of a $\mathrm{z}$-score transformation. In the parasympathetic pathway, the outputs $S_{i}$ from the EWN to the ciliary ganglion are as follows:

$$
S_{i}=f\left(-w_{i i} x_{i}-w_{j i} x_{j}+\beta_{i}\right)
$$

where $w_{j i}$ is the weight of the inhibitory synaptic connection from the $\mathrm{LC} j$ to EWN $i$ and $\beta_{i}$ represents all other inputs to EWN $i$. $f(x)=\tanh (x)+1$ is the activation function. The contralateral synaptic weights were set to $w_{12}=w_{21}=w_{c}$. In the sympathetic pathway, the inputs to the superior cervical ganglion are given by

$$
D_{i}=s_{i} x_{i}
$$

where $s_{i}$ denotes the synaptic weight of the LC connection to the superior cervical ganglion. Since, $D_{i}$ and $S_{i}$ correspond to dilator and sphincter muscle activation, respectively, the pupil diameter is proportional to

$$
P_{i}=D_{i}-S_{i}+P_{0},
$$

where $P_{0}$ is the base diameter of the pupil. The source code for simulating the $P_{i}$ can be found at the following address: https://github.com/SouNobukawa/neural_model_ for_controlling_pupil_diameter. In this study, we set $w_{11}=w_{22}=0.3, w_{12}=w_{21}=w_{c}, s_{i}=0.3, \beta_{1,2}=\beta, P_{0}=3.0$, and $L=1.5$. Figure 4 shows the typical time-series of variables of this model in the case with $\beta=2.0, b=4.8$. In comparison with experimentally measured pupil diameters shown in Figure 1, although detailed behaviors in time-series of $P_{i}$ were different, and complex and symmetric behaviors were similar. In the analysis for complexity and symmetricity, the evaluation duration was set to $10 \leq t \leq 300$ to involve dynamical intermittent behaviors such as the transition between symmetric and asymmetric behaviors. 


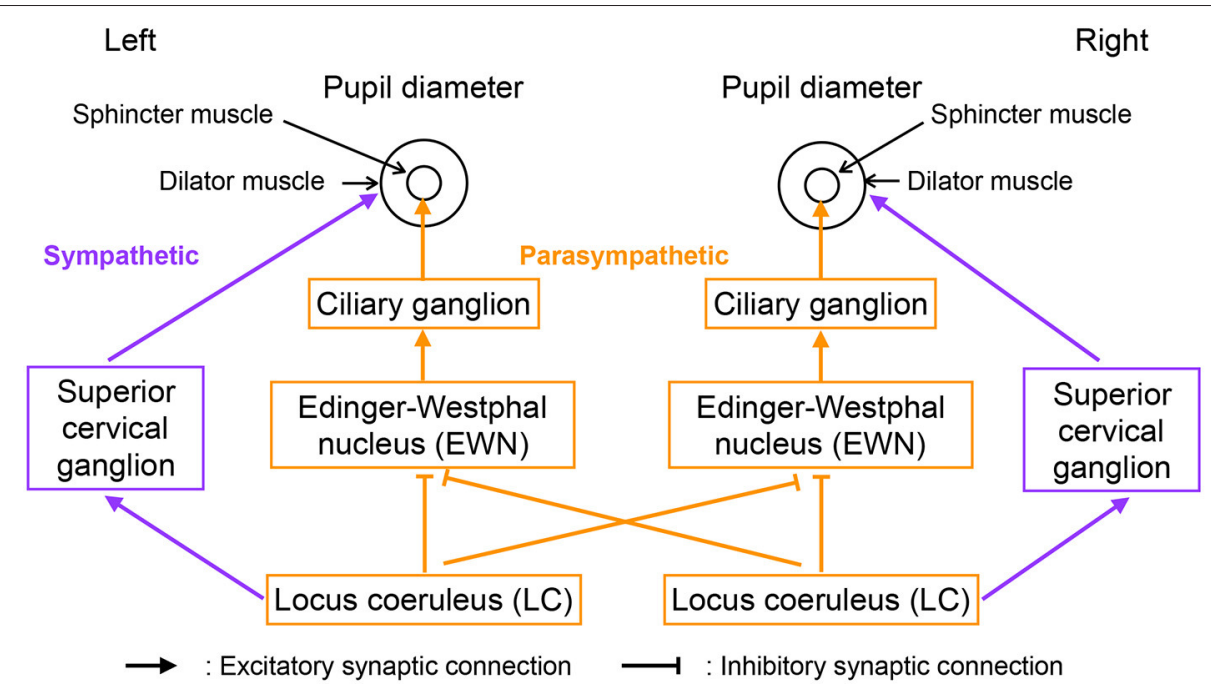

FIGURE 2 | Neural pathways controlling pupil diameter.

\section{A Coupled Lorenz model}

$$
\downarrow X_{i}
$$

B LC neural activity given by Eq.(10)

\section{Parasympathetic neural pathway}

C Output from the EWN to the ciliary ganglion given by Eq.(11)

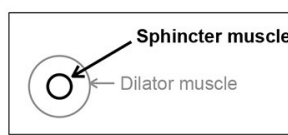

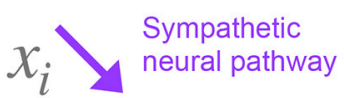

D Input to superior cervical ganglion given by Eq.(12)
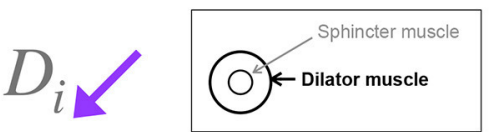

E Pupil diameters given by Eq.(13)<smiles>C1CCCCC1</smiles>

FIGURE 3 | Methodological flowchart for neural model of the controller of pupil diameter. (A) Coupled Lorenz model. (B) LC neural activity given by Equation (10). (C) Output from the EWN to the ciliary ganglion given by Equation (11). (D) Input to superior cervical ganglion given by Equation (12). (E) Pupil diameters given by Equation (13).

\subsection{Surrogate Data Analysis}

To investigate whether a non-linear dynamical process was involved in creating the observed pupil-diameter behavior, we generated surrogate data from a null hypothesis of no such involvement using the iterative amplitude-adjusted Fourier transformed (IAAFT) surrogate-data algorithm (Schreiber and Schmitz, 1996). The iteration number of the IAAFT procedure was 50. Ten IAAFT surrogate datasets were generated with different random seeds drawn from the pupil diameter measurements. The values of SampEn and TranEn calculated from these datasets were averaged and compared with the values derived from measurements.

\subsection{Statistical Analysis}

To compare the SampEn/TranEn of the measured time-series pupil data with those of the IAAFT surrogate data, we used a paired $t$-test. A two-tailed $\alpha$ level of 0.05 was adopted for statistical significance. If there was significant difference, then the time-series of the pupil-diameter was produced by a dynamical process and SampEn/TranEn could detect these dynamical properties.

To reveal relationships between the mean pupil diameter over time and SampEn or TranEn, a Gaussian-process regression (GPR), was used because the gaussian process is relatively robust for the data with inhomogeneous distribution (Williams and 


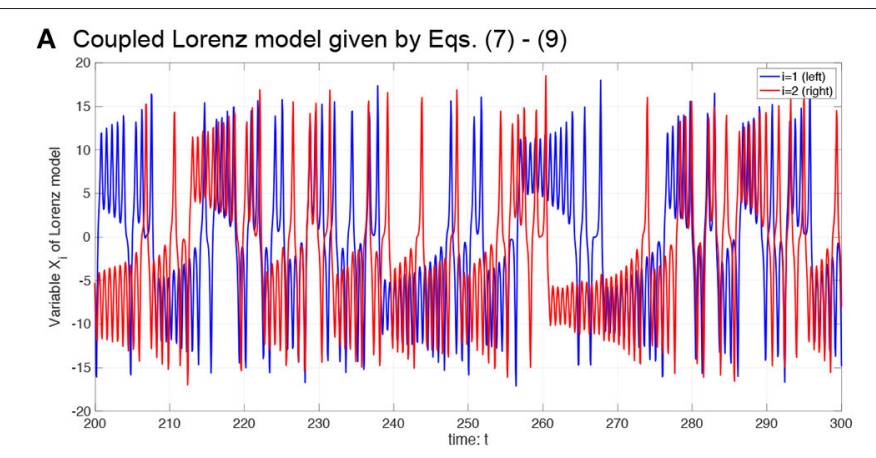

E Pupil diameters given by Eq.(13)

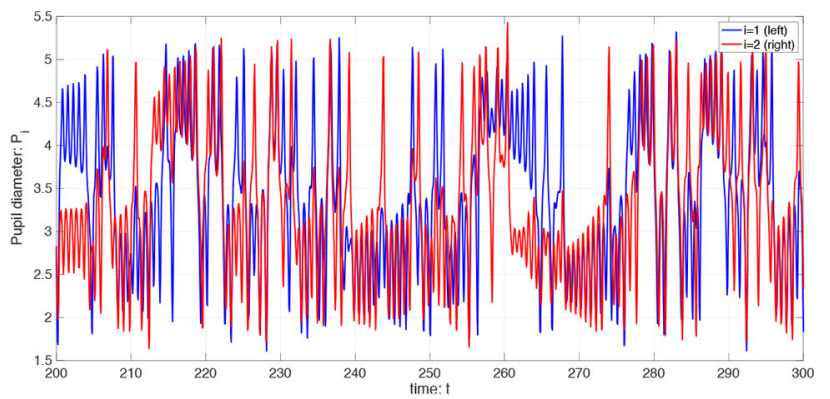

FIGURE 4 | Typical time-series of neural model of the controller of pupil diameter. (A) Variable $X_{i}$ in coupled Lorenz model. (B) Locus coeruleus (LC) activity $X_{i}$. (C) Output from the Edinger-Westphal nucleus (EWN) to the ciliary ganglion $S_{i}$. (D) Inputs to superior cervical ganglion $D_{i}$. (E) Pupil diameters $P_{i}$.

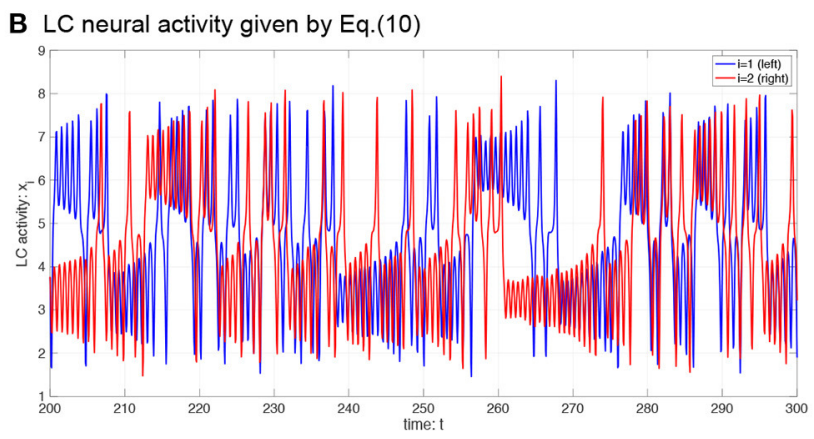

D Inputs to superior cervical ganglion given by Eq.(12)

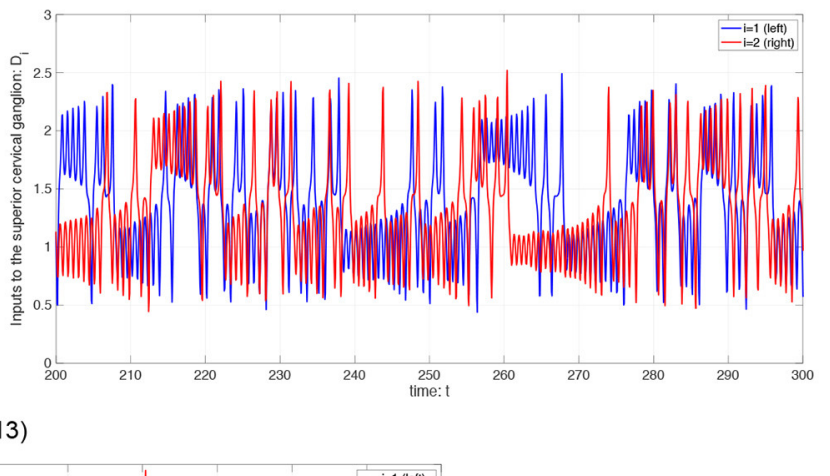

\section{.}

C Output from the EWN to the ciliary ganglion given by Eq.(11)

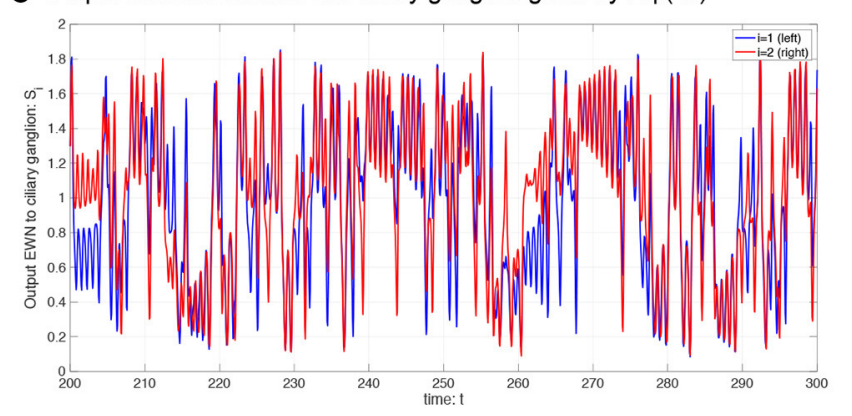

. 

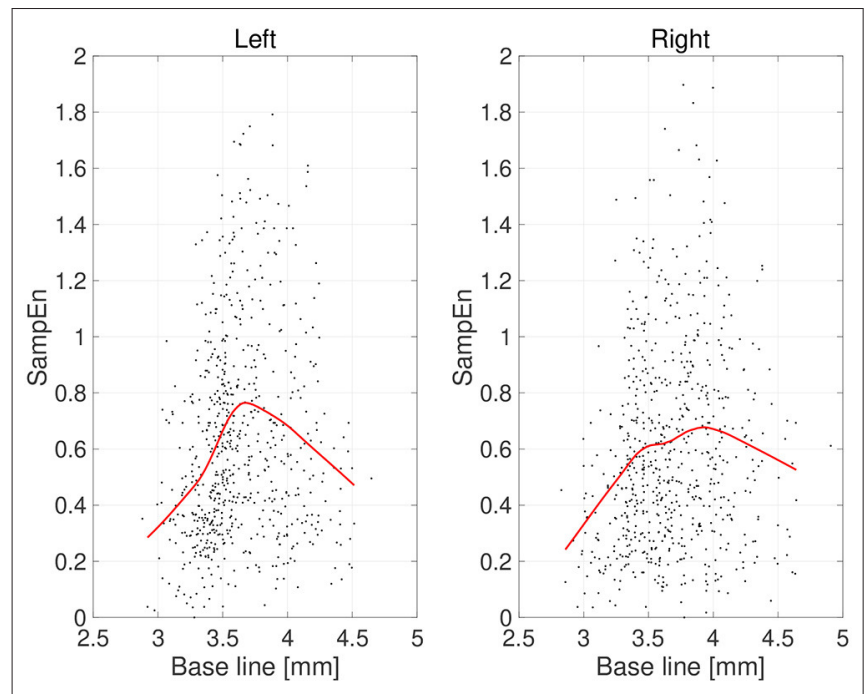

FIGURE 5 | Scatter plots of sample entropy (SampEn) vs. the mean values of pupil diameter (baseline) calculated for 2 -s epochs. Red line is a curve-fit conducted by a Gaussian process and moving average. An inverted-U dependence of SampEn on baseline is confirmed.

TABLE 1 | Surrogate-data analysis of the complexity of pupil-diameter time series.

\begin{tabular}{lccc}
\hline & Original & Surrogate data & $\boldsymbol{t}$-value (p-value) \\
\hline Left & $0.63(0.37)$ & $0.94(0.38)$ & $\mathbf{3 0 . 9 1 ( 0 . 0 0 )}$ \\
\hline Right & $0.60(0.37)$ & $0.92(0.37)$ & $\mathbf{3 0 . 8 6}(\mathbf{0 . 0 0})$ \\
\hline
\end{tabular}

Shown are the sample entropy (SampEn) of the original time series and of the corresponding iterative amplitude-adjusted Fourier transformed (IAAFT) surrogate timeseries. Mean (standard deviation) values of SampEn across epochs are presented. Paired t-tests show significant enhancement of SampEn by the surrogate process. For clarity, values with $p<0.05$ are shown in bold.

values of TranEn averaged across epochs in the original timeseries pupil data and in the corresponding IAAFT surrogate time-series. A significant enhancement of TranEn by the IAAFT process was confirmed. That is, like SampEn, TranEn reflects the contribution of a deterministic process.

\subsection{Simulation of Pupil Behavior}

We evaluated the complexity and symmetricity of changes in pupil diameter calculated from a neural model of pupil control. Figure 7A shows the dependence of SampEn and TranEn on the synaptic weight on the baseline activity of the LC (denoted by $b_{1,2}=b$ in Equation 10) in the case with the contralateral LC projections to the EWNs (denoted by $w_{12}=w_{21}=w_{c}=0.15$ in Equation 11). Here, the other input to EWN instead of input from LC is fixed to $\beta_{1}=\beta_{2}=\beta=2.0$. The results indicate that SampEn and TranEn exhibit an inverted U-shape against baseline $\mathrm{LC}$ activity. These peaks are SampEn $\approx 0.62$ and TranEn $\approx 0,22$ at baseline LC activity $b \approx 4.8$. While, the results in the case without the contralateral LC projections $\left(w_{c}=0\right)$ are shown in Figure 7B. SampEn does not exhibit a definite peak; the peak of TranEn becomes smaller in comparison with the case with contralateral LC projections of Figure 7A.

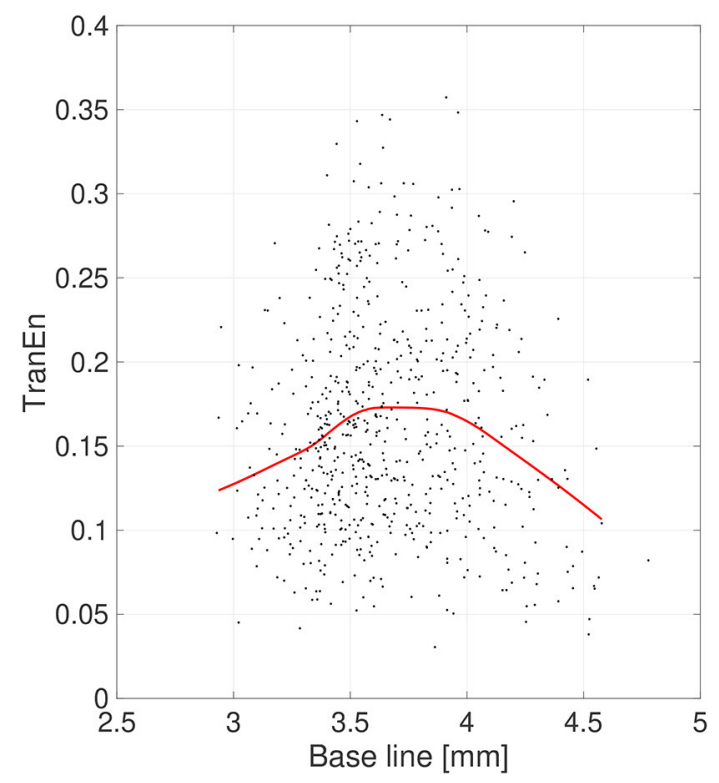

FIGURE 6 | Scatter plots of transfer entropy (TranEn) vs. the mean values of pupil diameter (baseline) calculated for 2 -s epochs. Here, the baseline values are averaged between right and left pupils and the TranEn values are averaged between the right-to-left and left-to-right cases. Red line is a curve-fit conducted by a Gaussian process and moving average. An inverted- $U$ dependence of TranEn on baseline is confirmed.

TABLE 2 | Surrogate-data analysis of the symmetricity of pupil-diameter time series.

\begin{tabular}{lcc}
\hline Original & Surrogate data & $\boldsymbol{t}$-value (p-value) \\
\hline $0.16(0.06)$ & $0.18(0.05)$ & $\mathbf{1 1 . 4}(\mathbf{0 . 0 0})$ \\
\hline
\end{tabular}

Shown are the transfer entropy (TranEn) of the original time series and of the corresponding IAAFT surrogate time-series. Mean (standard deviation) values of TranEn across epochs are presented. Paired t-tests show significant enhancement of TranEn by the surrogate process. For clarity, values with $p<0.05$ are shown in bold.

Furthermore, we investigate the influence of $\beta$ on the profile of SampEn and TranEn under the contralateral LC projections to the EWNs. Figure 8 shows dependence of SampEn in left and right eyes on the baseline activity of LC in the case with $\beta_{1}=\beta_{2}=\beta=2.0,3.5,5.0$ under the contralateral projection from the LC to EWN $w_{12}=w_{21}=w_{c}=0.15$ and corresponding dependence of TranEn. Here, the $\beta=2.0$ case corresponds to the result of Figure 7A. The peaks of SampEn and TranEn shift to the region of large $b$, increasing with $\beta$.

\section{DISCUSSION}

In this study, we evaluated the complexity and symmetricity of the autonomous variations in pupil diameter in healthy participants. First, surrogate data analysis revealed that the complexity and symmetricity of changes in pupil diameter reflect the presence of a non-linear deterministic process. Because pupil diameters are controlled by parasympathetic and sympathetic pathways, this result implies that these complexity 

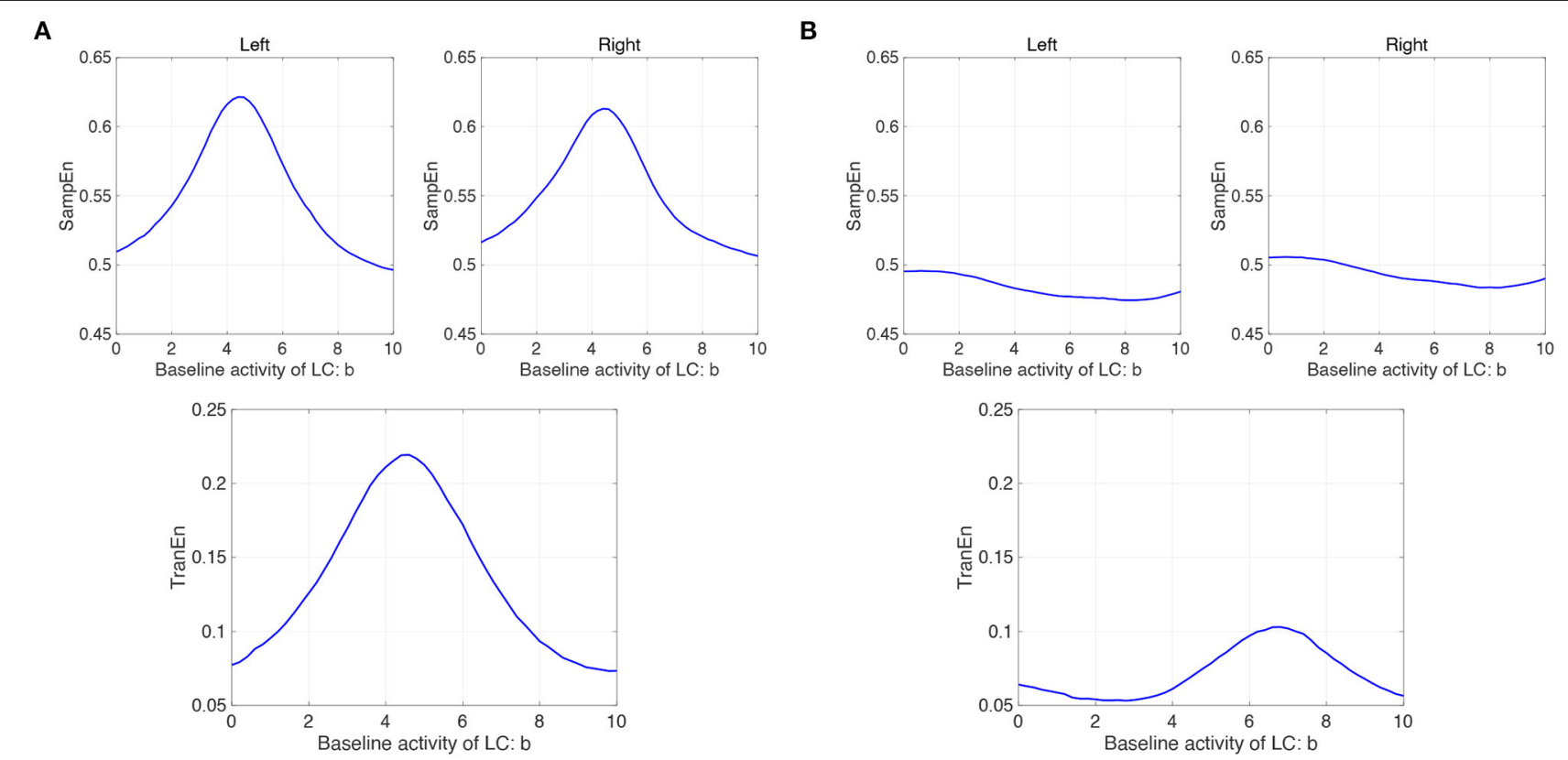

FIGURE 7 | Modeling results: (A) (Upper parts) Dependence of SampEn in left and right eyes on the baseline activity of the locus coeruleus (LC) (denoted by $b_{1,2}=b$ in Equation 10) in the case with the contralateral projection from the LC to the Edinger-Westphal nucleus (EWN) (denoted by $w_{12}=W_{21}=w_{C}=0.15$ in Equation 11 ). (Lower part) Corresponding dependence of TranEn. SampEn and TranEn exhibit an inverted-U shape against baseline LC activity. (B) (Upper parts) Dependence of SampEn in left and right eyes on the baseline activity of the LC in the case without the contralateral projection from the LC to the EWN $\left(w_{12}=w_{21}=w_{C}=0\right.$ in Equation 11). (Lower part) Corresponding dependence of TranEn. SampEn does not exhibit definite peak; the peak of TranEn becomes smaller in comparison with the case with contralateral LC projections.

and symmetricity measures are physiologically inherent in the neural dynamics. Second, we investigated the relationships between the pupil-diameter baselines, which reflect tonic LC activity, and both the complexity and the symmetricity. The results indicated that both measures had a unimodal maximum at intermediate pupil diameters. Third, the simulation results from the neural model of pupil control showed that the nonlinear threshold characteristics of the EWNs induce the observed inverted U-shaped profiles; this tendency is enhanced if the contralateral synaptic projections from the LC to the EWNs are above a certain strength.

The complexity and temporal patterns of pupil-diameter changes reflect LC activity, sleepiness, cognitive processes, autonomic nerve activity, and psychiatric disorders (Usui and Stark, 1982; Schumann et al., 2015; Artoni et al., 2019; Nakamura et al., 2019; Piu et al., 2019). Sleepiness in particular decreases the temporal complexity and determinicity of pupil diameter (Piu et al., 2019). Nakamura et al. reported that at the discrimination stage of the cognitive process, the complexity of pupil-diameter changes is reduced and pupil dilation occurs (Nakamura et al., 2019). Artoni et al. demonstrated that convolutional neural networks can detect disease-specific temporal patterns in the fluctuations of pupil diameter, a result obtained from mouse models of ASD. (Artoni et al., 2019). Moreover, studies on asymmetricity in pupil diameter have found that dilation appears strongly and independently in either pupil as a reaction to cognitive load (Kim et al., 1998; Kahya et al., 2018). Wahn et al. reported that the asymmetricity of pupil diameters also correlates with attentional load (Wahn et al., 2017). The above findings show that complexity and asymmetricity reflect a broad range of internal neural processes, thereby supporting our results on the emergence of specific complexity and symmetricity profiles against pupil baseline diameter, the latter correlating with LC activity.

The inverted $U$-shaped profiles of complexity and symmetricity against baseline activity require an explanation. This relationship was first reported by Usui and Stark (1982). Through simulations based on a neuronal model of the system controlling pupil diameter, these researchers found that the inverted U-shape is induced by a non-linear threshold characteristic of pupil dilation vs. the neural input signals to the system (Usui and Stark, 1982). In our model, this non-linear threshold characteristic corresponds to the EWN activation function $f(x)$. More concretely, at low LC baseline activity, the output of the EWNs $S_{i}$ converges to $\approx 2.0$ and its variability is small. With increasing LC activity, $S_{i}$ will be in the vicinity of the threshold of $f(x)$ due to the inhibitory connections $w_{i j}$ from the LC to the EWNs. In this state, the pupil diameters are driven by both dilator commands $D_{i}$ and sphincter commands $S_{i}$, resulting in large variability and peak complexity. Moreover, with appreciable synaptic weight at the contralateral connection to the EWNs, the contralateral LC activity $x_{i}$ affects $S_{i}$, enhancing the peak of complexity. With further increases in LC activity, $S_{i}$ is $\approx 0.0$, with the result that the pupil diameter is driven only by 

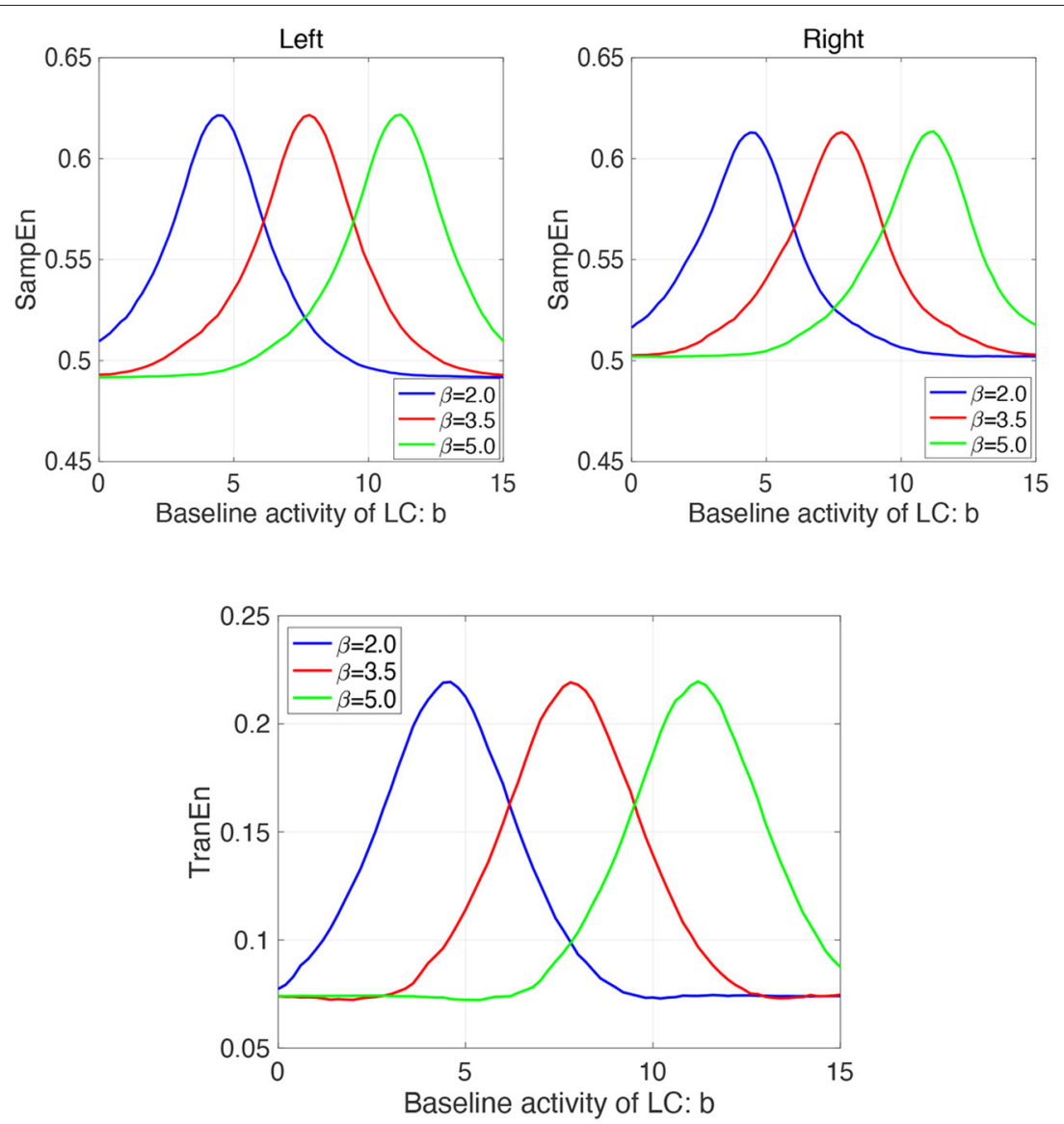

FIGURE 8 | Modeling Results: (Upper parts) Dependence of SampEn in left and right eyes on the baseline activity of LC in the case with the other input to EWN instead of input from $L C \beta_{1}=\beta_{2}=\beta=2.0,3.5,5.0$ under the contralateral projection from the LC to $E W N w_{12}=w_{21}=w_{C}=0.15$. (Lower part) Corresponding dependence of TranEn. The peaks of SampEn and TranEn shift to the region of large $b$, increasing with $\beta$.

$D_{i}$, resulting in decreased complexity. The inverted U-shape of symmetricity plotted against baseline LC activity is also induced by the EWN activation function. In the case of small LC baseline activity, common LC inputs to EWN through the contralateral synapses are weak, resulting in low symmetricity between the two EWNs $S_{1,2}$ and thus between the two pupils. With increasing LC baseline activity, the LC drives the contralateral outputs to EWN $S_{1,2}$ more strongly. The resulting common input to each side of the EWN enhances the symmetricity. With still greater LC activity, $S_{i}$ is $\approx 0.0$, and the pupils are driven only by the sympathetic pathway, which has no contralateral projections. Here, in larger other input to EWN instead of LC (represented by $\beta_{i}$ in Equation 11), greater LC activity is needed to realize $S_{i} \approx 0.0$. Therefore, the symmetricity decreases.

The advantages of evaluating complexity and symmetricity as SampEn and TranEn, respectively, requires further discussion. Conventional evaluation methods for pupil complexity and symmetricity utilize temporal standard deviation, Shannon entropy, and the difference between right and left pupil diameters (Usui and Stark, 1982; Poynter, 2017; Wahn et al., 2017; Piu et al., 2019). Moreover, Piu et al. showed that the combination of complexity estimation by Shannon entropy and determinism is an effective diagnostic tool for identifying the states of neural systems (Piu et al., 2019). The importance of determinism for evaluation is supported by the fact that changes in pupil diameter are produced by multiple nonlinear neural pathways (Usui and Stark, 1982; Liu et al., 2017). However, the temporal standard deviation, Shannon entropy, and difference in pupil diameters cannot capture contributions from deterministic processes (Theiler et al., 1991; Schreiber and Schmitz, 1996). The SampEn and TranEn measures utilized in this study can precisely detect the contribution of determinism to pupil diameter changes, in addition to permitting evaluations of complexity and symmetricity (see Tables 1, 2). Therefore, it can be assumed that evaluation by SampEn and TranEn is a useful method for estimating the states of neural systems and deficits in neural function.

This study has several limitations. We revealed the typical profiles of complexity and symmetricity for autonomous pupillary changes. However, the nature of these change profiles in the presence of cognitive tasks and attentional loads remains unclear. Moreover, we only studied healthy participants in this study. It is imperative to measure the corresponding profiles in patients with psychiatric disorders involving deficits of attention 
and arousal, and those involving imbalances in explorationexploitation, such as schizophrenia, ASD, and ADHD. In the model-based analysis, we modeled the LC activity as two neural populations sustaining weakly synchronized, chaotic oscillations. Recent progress in understanding the neural activity of the LC revealed that this activity exhibits complex spatio-temporal behaviors among multiple neural populations (Totah et al., 2019). However, the population size used in the present study is too small to describe these spatio-temporal behaviors. Thus, it will be necessary to increase the size of the neural populations or utilize cellular non-linear networks (Arena et al., 2005; Bucolo et al., 2008) and spiking neural networks (Nobukawa et al., 2019, 2020 ) with high physiological validity. We are currently planning to address these queries in future works.

\section{CONCLUSIONS}

In this study, we characterized the typical relationships between baseline activity, complexity, and symmetricity for resting-state changes in pupil diameter using the measures SampEn and TranEn by analyzing pupil diameters and constructing a model controller of pupil diameter. Our proposed evaluation method and our findings may find application in the development of pupillometric estimation and diagnostic tools for application to healthy brain states, their functions, and to psychiatric disorders.

\section{DATA AVAILABILITY STATEMENT}

The datasets presented in this article are not readily available because the datasets generated for this study will not be made

\section{REFERENCES}

Anderson, C. J., and Colombo, J. (2009). Larger tonic pupil size in young children with autism spectrum disorder. Dev. Psychobiol. 51, 207-211. doi: $10.1002 /$ dev.20352

Arena, P., Bucolo, M., Fazzino, S., Fortuna, L., and Frasca, M. (2005). The CNN paradigm: shapes and complexity. Int. J. Bifurcat. Chaos 15, 2063-2090. doi: 10.1142/S0218127405013307

Artoni, P., Piffer, A., Vinci, V., LeBlanc, J., Nelson, C. A., Hensch, T. K., et al. (2019). Deep learning of spontaneous arousal fluctuations detects early cholinergic defects across neurodevelopmental mouse models and patients. Proc. Natl. Acad. Sci. U.S.A. 117, 23298-23303. doi: 10.1073/pnas.1820847116

Aston-Jones, G., and Cohen, J. D. (2005). An integrative theory of locus coeruleusnorepinephrine function: adaptive gain and optimal performance. Annu. Rev. Neurosci. 28, 403-450. doi: 10.1146/annurev.neuro.28.061604.135709

Bast, N., Banaschewski, T., Dziobek, I., Brandeis, D., Poustka, L., and Freitag, C. M. (2019). Pupil dilation progression modulates aberrant social cognition in autism spectrum disorder. Autism Res. 12, 1680-1692. doi: 10.1002/aur.2178

Brainard, D. H. (1997). The psychophysics toolbox. Spatial Vision 10, 433-436.

Breen, L. A., Burde, R. M., and Loewy, A. D. (1983). Brainstem connections to the edinger-westphal nucleus of the cat: a retrograde tracer study. Brain Res. 261, 303-306. doi: 10.1016/0006-8993(83)90633-9

Bucolo, M., Buscarino, A., Fortuna, L., Frasca, M., and Xibilia, M. G. (2008). From dynamical emerging patterns to patterns in visual art. Int. J. Bifurcat. Chaos 18, 51-81. doi: $10.1142 / S 021812740802015 \mathrm{X}$

Costa, M., Goldberger, A. L., and Peng, C. K. (2002). Multiscale entropy analysis of complex physiologic time series. Phys. Rev. Lett. 89:068102. doi: 10.1103/PhysRevLett.89.068102 publicly available because the informed consent did not include the declaration regarding publicity of clinical data. Requests to access the datasets should be directed to Sou Nobukawa, nobukawa@cs.it-chiba.ac.jp.

\section{ETHICS STATEMENT}

The studies involving human participants were reviewed and approved by Ethics Committee of Showa University. The patients/participants provided their written informed consent to participate in this study.

\section{AUTHOR CONTRIBUTIONS}

SN, AS, TTaka, and ST conceived the methods. SN, AS, TTaka, $\mathrm{MK}$, and ST analyzed and discussed the results. SN, AS, TTaka, and ST wrote the main manuscript and prepared all figures. AS, TTake, HO, AI, NK, and ST conducted the experiments. All authors contributed to manuscript revision and have read and approved the submitted version.

\section{FUNDING}

This study was supported by the Joint Usage/Research Program of Medical Institute of Developmental Disabilities Research, Showa University. It was also supported by JSPS KAKENHI for [Research Activity Start-up (Grant Number 19K23395) and Scientific Research (C) (Grant Number 20K03490) to AS] and [Scientific Research (C) (Grant Number 20K07928) to $\mathrm{ST}$ ].

Ebitz, R. B., Pearson, J. M., and Platt, M. L. (2014). Pupil size and social vigilance in rhesus macaques. Front. Neurosci. 8:100. doi: 10.3389/fnins.2014.00100

Ego-Stengel, V., Bringuier, V., and Shulz, D. (2002). Noradrenergic modulation of functional selectivity in the cat visual cortex: an in vivo extracellular and intracellular study. Neuroscience 111, 275-289. doi: 10.1016/s0306-4522(02)00011-8

Eldar, E., Cohen, J. D., anf Niv, Y. (2013). The effects of neural gain on attention and learning. Nat. Neurosci. 16, 1146-1153. doi: 10.1038/nn.3428

Gilzenrat, M., Cohen, J., Rajkowski, J., and Aston-Jones, G. (2003). "Pupil dynamics predict changes in task engagement mediated by locus coeruleus," in Society for Neuroscience Abstracts.

Goldberger, A. L., Amaral, L. A., Glass, L., Hausdorff, J. M., Ivanov, P. C., Mark, R. G., et al. (2000). PhysioBank, PhysioToolKit, and PhysioNet: components of a new research resource for complex physiologic signals. Circulation 101, E215-E220. doi: 10.1161/01.cir.101.23.e215

Gotham, K. O., Siegle, G. J., Han, G. T., Tomarken, A. J., Crist, R. N., Simon, D. M., et al. (2018) Pupil response to social-emotional material is associated with rumination and depressive symptoms in adults with autism spectrum disorder. PLoS ONE 13:e0200340. doi: 10.1371/journal.pone.0200340

Hirata, A., Aguilar, J., and Castro-Alamancos, M. A. (2006). Noradrenergic activation amplifies bottom-up and top-down signal-to-noise ratios in sensory thalamus. J. Neurosci. 26, 4426-4436. doi: 10.1523/JNEUROSCI.5298-05.2006

Hong, L., Walz, J. M., and Sajda, P. (2014). Your eyes give you away: prestimulus changes in pupil diameter correlate with poststimulus taskrelated EEG dynamics. PLoS ONE 9:e91321. doi: 10.1371/journal.pone.00 91321

Johansson, B., and Balkenius, C. (2018). A computational model of pupil dilation. Connect. Sci. 30, 5-19. doi: 10.1080/09540091.2016.1271401 
Joshi, S., Li, Y., Kalwani, R. M., and Gold, J. I. (2016). Relationships between pupil diameter and neuronal activity in the locus coeruleus, colliculi, and cingulate cortex. Neuron 89, 221-234. doi: 10.1016/j.neuron.2015.11.028

Kahya, M., Wood, T. A., Sosnoff, J. J., and Devos, H. (2018). Increased postural demand is associated with greater cognitive workload in healthy young adults: a pupillometry study. Front. Hum. Neurosci. 12:288. doi: 10.3389/fnhum.2018.00288

Kim, M., Barrett, A., and Heilman, K. (1998). Lateral asymmetries of pupillary responses. Cortex 34, 753-762.

Liu, Y., Rodenkirch, C., Moskowitz, N., Schriver, B., and Wang, Q. (2017). Dynamic lateralization of pupil dilation evoked by locus coeruleus activation results from sympathetic, not parasympathetic, contributions. Cell Rep. 20, 3099-3112. doi: 10.1016/j.celrep.2017.08.094

Longtin, A., and Milton, J. G. (1989). Modelling autonomous oscillations in the human pupil light reflex using non-linear delay-differential equations. Bull. Math. Biol. 51, 605-624.

Martineau, J., Hernandez, N., Hiebel, L., Roché, L., Metzger, A., and BonnetBrilhault, F. (2011). Can pupil size and pupil responses during visual scanning contribute to the diagnosis of autism spectrum disorder in children? J. Psychiatr. Res. 45, 1077-1082. doi: 10.1016/j.jpsychires.2011.01.008

Martins, A. R. O., and Froemke, R. C. (2015). Coordinated forms of noradrenergic plasticity in the locus coeruleus and primary auditory cortex. Nat. Neurosci. 18, 1483-1492. doi: 10.1038/nn.4090

McCormick, D., and Pape, H. (1990). Noradrenergic and serotonergic modulation of a hyperpolarization-activated cation current in thalamic relay neurones. $J$. Physiol. 431, 319-342. doi: 10.1113/jphysiol.1990.sp018332

McGinley, M. J., Vinck, M., Reimer, J., Batista-Brito, R., Zagha, E., Cadwell, C. R., et al. (2015) Waking state: rapid variations modulate neural and behavioral responses. Neuron 87, 1143-1161. doi: 10.1016/j.neuron.2015.09.012

Moxon, K. A., Devilbiss, D. M., Chapin, J. K., and Waterhouse, B. D. (2007). Influence of norepinephrine on somatosensory neuronal responses in the rat thalamus: a combined modeling and in vivo multi-channel, multi-neuron recording study. Brain Res. 1147, 105-123. doi: 10.1016/j.brainres.2007.02.006

Nakamura, N. H., Fukunaga, M., and Oku, Y. (2019). Respiratory fluctuations in pupil diameter are not maintained during cognitive tasks. Respir. Physiol. Neurobiol. 265, 68-75. doi: 10.1016/j.resp.2018.07.005

Nassar, M. R., Rumsey, K. M., Wilson, R. C., Parikh, K., Heasly, B., and Gold, J. I. (2012). Rational regulation of learning dynamics by pupil-linked arousal systems. Nat. Neurosci. 15, 1040-1046. doi: 10.1038/nn.3130

Niso, G., Bruña, R., Pereda, E., Gutiérrez, R., Bajo, R., Maestú, F., et al. (2013). HERMES: towards an integrated toolbox to characterize functional and effective brain connectivity. Neuroinformatics 11, 405-434. doi: 10.1007/s12021-0139186-1

Nobukawa, S., Nishimura, H., Wagatsuma, N., Ando, S., and Yamanishi, T. (2020). Long-tailed characteristic of spiking pattern alternation induced by lognormal excitatory synaptic distribution. IEEE Trans. Neural Netw. Learn. Syst. doi: 10.1109/TNNLS.2020.3015208. [Epub ahead of print].

Nobukawa, S., Nishimura, H., and Yamanishi, T. (2019). Temporal-specific complexity of spiking patterns in spontaneous activity induced by a dual complex network structure. Sci. Rep. 9:12749. doi: 10.1038/s41598-019-49286-8

Pamplona, V. F., Oliveira, M. M., and Baranoski, G. V. (2009). Photorealistic models for pupil light reflex and iridal pattern deformation. ACM Trans. Graph. 28:106. doi: 10.1145/1559755.1559763

Pelli, D. G. (1997). The videotoolbox software for visual psychophysics: Transforming numbers into movies. Spatial Vision 10, 437-442.

Piu, P., Serchi, V., Rosini, F., and Rufa, A. (2019). A cross-recurrence analysis of the pupil size fluctuations in steady scotopic conditions. Front. Neurosci. 13:407. doi: 10.3389/fnins.2019.00407

Poynter, W. D. (2017). Pupil-size asymmetry is a physiologic trait related to gender, attentional function, and personality. Laterality 22, 654-670. doi: 10.1080/1357650X.2016.1268147
Rajkowski, J. (1993). “Correlations between locus coeruleus (LC) neural activity, pupil diameter and behavior in monkey support a role of LC in attention," in Soc. Neurosc., Abstract (Washington, DC).

Reddy, L. F., Reavis, E. A., Wynn, J. K., and Green, M. F. (2018). Pupillary responses to a cognitive effort task in schizophrenia. Schizophrenia Res. 199, 53-57. doi: 10.1016/j.schres.2018.03.005

Reimer, J., Froudarakis, E., Cadwell, C. R., Yatsenko, D., Denfield, G. H., and Tolias, A. S. (2014). Pupil fluctuations track fast switching of cortical states during quiet wakefulness. Neuron 84, 355-362. doi: 10.1016/j.neuron.2014.09.033

Richman, J. S., and Moorman, J. R. (2000). Physiological time-series analysis using approximate entropy and sample entropy. Am. J. Physiol. Heart Circ. Physiol. 278, H2039-H2049. doi: 10.1152/ajpheart.2000.278.6.H2039

Schreiber, T. (2000). Measuring information transfer. Phys. Rev. Lett. 85:461. doi: 10.1103/PhysRevLett.85.461

Schreiber, T., and Schmitz, A. (1996). Improved surrogate data for nonlinearity tests. Phys. Rev. Lett. 77:635.

Schumann, A., Kralisch, C., and Bär, K. J. (2015). "Spectral decomposition of pupillary unrest using wavelet entropy," in 2015 37th Annual International Conference of the IEEE Engineering in Medicine and Biology Society (EMBC) (Milan: IEEE), 6154-6157.

Szabadi, E. (2018). Functional organization of the sympathetic pathways controlling the pupil: light-inhibited and light-stimulated pathways. Front. Neurol. 9:1069. doi: 10.3389/fneur.2018.01069

Thakkar, K. N., Brascamp, J. W., Ghermezi, L., Fifer, K., Schall, J. D., and Park, S. (2018). Reduced pupil dilation during action preparation in schizophrenia. Int. J. Psychophysiol. 128, 111-118. doi: 10.1016/j.ijpsycho.2018.03.012

Theiler, J., Galdrikian, B., Longtinm, A., Eubank, S., and Farmer, J. D. (1991). Testing for Nonlinearity in Time Series: The Method of Surrogate Data. Technical report. Los Alamos National Lab.

Totah, N. K., Logothetis, N. K., and Eschenko, O. (2019). Noradrenergic ensemblebased modulation of cognition over multiple timescales. Brain Res. 1709, 50-66. doi: 10.1016/j.brainres.2018.12.031

Usui, S., and Stark, L. (1982). A model for nonlinear stochastic behavior of the pupil. Biol. Cybernet. 45, 13-21.

van der Wel, P., and van Steenbergen, H. (2018). Pupil dilation as an index of effort in cognitive control tasks: a review. Psychon. Bull. Rev. 25, 2005-2015. doi: 10.3758/s13423-018-1432-y

Wahn, B., Ferris, D. P., Hairston, W. D., and König, P. (2017). Pupil size asymmetries are modulated by an interaction between attentional load and task experience. bioRxiv [Preprint] 137893. doi: 10.1101/137893

Wainstein, G., Rojas-Líbano, D., Crossley, N., Carrasco, X., Aboitiz, F., and Ossandón, T. (2017). Pupil size tracks attentional performance in attention-deficit/hyperactivity disorder. Sci. Rep. 7:8228. doi: 10.1038/s41598-017-08246-w

Watson, A. B., and Yellott, J. I. (2012). A unified formula for light-adapted pupil size. J. Vis. 12:12. doi: 10.1167/12.10.12

Wekselblatt, J. B., and Niell, C. M. (2015). Behavioral state-getting "in the zone". Neuron 87, 7-9. doi: 10.1016/j.neuron.2015.06.020

Williams, C. K., and Rasmussen, C. E. (2006). Gaussian Processes for Machine Learning, Vol. 2. Cambridge, MA: MIT Press.

Conflict of Interest: The authors declare that the research was conducted in the absence of any commercial or financial relationships that could be construed as a potential conflict of interest.

Copyright (c) 2021 Nobukawa, Shirama, Takahashi, Takeda, Ohta, Kikuchi, Iwanami, Kato and Toda. This is an open-access article distributed under the terms of the Creative Commons Attribution License (CC BY). The use, distribution or reproduction in other forums is permitted, provided the original author(s) and the copyright owner(s) are credited and that the original publication in this journal is cited, in accordance with accepted academic practice. No use, distribution or reproduction is permitted which does not comply with these terms. 